\title{
The Effect of an Encapsulated Specimen's Mounting Material on Its Rockwell Hardness Test Results
}

\author{
George K. Abraham IV
}

Received: 7 May 2013/Revised: 11 October 2013/Accepted: 15 October 2013/Published online: 24 October 2013

(C) Springer Science+Business Media New York and ASM International 2013

\begin{abstract}
This study addresses the affect of mounting material used with an encapsulated metallographic specimen on its Rockwell hardness test results. Thirty-five specimens were sectioned from a grade 1144 medium carbon steel rod as the experimental material. Thirty of the sections were encapsulated in six different mounting materials (the test groups), and five were left unencapsulated (the control group). Each specimen was ground and polished using the same metallographic preparation procedure, and then hardness tests were performed using the Rockwell hardness C scale. The repeatability $\left(r_{\mathrm{PB}}\right)$ value, from the ASTM International Inter-Laboratory Study to Establish Precision Statements for ASTM E18, was used to compare the hardness test results of the encapsulated test groups to the results of the unencapsulated control group. Using that study, if the average hardness of a test group fell within $\pm r_{\mathrm{PB}}$ of the control group, there would be approximately a $95 \%$ probability that it was equivalent to the control group. Five out of the six test groups (all except transparent thermoplastic) had average hardness values that fell within $\pm r_{\mathrm{PB}}$ of the control group average. Four of the six test groups (all except green phenolic and transparent thermoplastic) had standard deviations that were less than $r_{\mathrm{PB}}$. With such results, green phenolic and transparent thermoplastic are not suitable mounting materials for Rockwell hardness testing.
\end{abstract}

This article is an invited paper selected from presentations at the 2013 Quantitative Metallography Conference and Exposition, held April 4-5, 2013, in San Antonio, Texas, and has been expanded from the original presentation.

G. K. Abraham IV ( $\square)$

Allied High Tech Products, Inc., Rancho Dominguez, CA, USA

e-mail: gkabraham@alliedhightech.com
Keywords Rockwell hardness · Mounting material · Encapsulation $\cdot$ Repeatability

\section{Introduction}

A Rockwell hardness indentation test consists of three main steps: load applied, load removed, and determination of the hardness from measurements of the resulting indentation. The difference between these depths $(h)$ is used to calculate the Rockwell hardness value [1]. Using the Rockwell hardness C scale (HRC), a difference in $h$ of only $10 \mu \mathrm{m}$ will produce a hardness value difference of 5 HRC. This illustrates the importance of preventing the test specimen from shifting and to produce a uniform test surface. When performing a Rockwell hardness test, a commonly accepted practice is to avoid encapsulating specimens in mounting materials for metallographic preparation, instead testing each specimen in its unencapsulated state [2]. The mounting material could distort and cause the specimen to shift while load is applied during a hardness test. Hardness test results for encapsulated specimens will theoretically become less repeatable and less reliable under higher applied loads and lower mounting material hardness values.

Section 5.5.1 of the ASTM International Standard Test Methods for Rockwell Hardness of Metallic Materials (ASTM E18-11) states that "common specimen support anvils should have a minimum hardness of 58 HRC" when performing Rockwell hardness tests [1]. For encapsulated specimens, the support anvil would be defined as the mounting material. Most metallographic mounting materials are types of polymers, for which hardness values are measured using a Shore durometer scale, conforming to the ASTM International Standard Test Method for Rubber 
Property-Durometer Hardness (ASTM D2240-05) [3]. Each durometer type is "specifically designed to determine a specific scale, and the scale does not exist separately from the durometer" [4]. This means that conversions between two different durometer scales, or a durometer scale and another hardness test scale, are not reliable. Hardness values of mounting materials are therefore assumed to be lower than $58 \mathrm{HRC}$, so an encapsulated specimen would seem to not conform to ASTM E18-11 or commonly accepted practices.

The ASTM International Inter-Laboratory Study to Establish Precision Statements for ASTM E18 (ASTM RR:E28-1021) provided results of a Rockwell hardness precision and bias study for test blocks of varying hardnesses [5]. That study was performed to determine what would be considered normal variations in hardness test values by a user. Repeatability $\left(r_{\mathrm{PB}}\right)$ values were found for various test blocks, which represent the typical amounts of variation between test results for the same sample material, using the same hardness tester on the same day, and performed by the same operator. If the measurement difference between test results is less than $r_{\mathrm{PB}}$, then there is approximately a $95 \%$ probability that the results are equivalent. For this author's study, $r_{\mathrm{PB}}$ was used to compare each mounting material group to an unencapsulated control group, to determine whether a mounting material is not supporting its specimen adequately.

\section{Materials and Methods}

A 25.4-mm diameter grade 1144 steel rod was acquired from a commercial source; it was chosen due to its relatively high hardness without further heat treating, meeting the ASTM International Standards Specification for Steel Bars, Carbon and Alloy, Hot Wrought, General Requirements for (ASTM A29), Specification for Steel Bar, Carbon and Alloy, Cold-Finished (ASTM A108), and Specification for Cold Drawn, Stress Relieved Carbon Steel Bars Subject to Mechanical Property Requirements (ASTM A311), with a stated hardness of 23 HRC [6].

The steel rod was sectioned into 35 pieces using a high speed abrasive cut-off saw. A rubber bonded aluminum oxide blade was used since it is a standard blade type for sectioning ferrous metals. Thirty specimens were sectioned to about 7-mm thick; these were to be encapsulated in six different mounting materials. A Rockwell indentation on a 23 HRC material would be expected to produce a value of $h$ of $0.154 \mathrm{~mm}$, using the equation found in Sect. 5.2.1.1 of ASTM E18-11 [1]. Section 6.3 of ASTM E18-11 recommends that the test piece "thickness should exceed 10 times the depth of indentation", which would require at least a 1.54-mm thick specimen. The specimen should actually be slightly thicker than that, due to the indentation depth during the application of the total force being an unknown value greater than $h$, which is shown in Fig. 1 of ASTM E18-11. Thus, a thickness of $7 \mathrm{~mm}$ for the specimens in this study more than ensured that the zone of deformation from indentations would not extend through the specimens into the respective mounting materials. This is important because this study is addressing the hardness values of the steel pieces and not the hardness values of the mounting materials. Five specimens were sectioned to about $25-\mathrm{mm}$ thick; these were to remain unencapsulated as the control group. They needed to be thicker than the other sections for ease of handling during the grinding and polishing steps.

The mounting materials used in this study include green phenolic (with wood filler), orange phenolic (with melamine filler), black glass-filled epoxy, transparent thermoplastic, QuickCure acrylic, and EpoxySet epoxy [7]. These mounting materials were chosen as a representative group of common mounting materials available from metallographic consumable suppliers. Using the recommended equipment and curing schedules for each mounting material, five of the 7-mm thick specimens were encapsulated in each of the six mounting materials using 38.1-mm diameter molds, producing 30 encapsulated specimens. The five control group specimens were not encapsulated.

Each specimen was ground using a series of silicon carbide abrasive discs, followed by polishing with $3-\mu \mathrm{m}$ polycrystalline diamond suspension on a DiaMat polishing cloth, which is a cloth composed of woven wool fibers [8]. The bottoms of the mounts and control specimens were all ground flat to provide adequate registration with the hardness tester anvil. After each step, the samples were cleaned with soap and water, and then rinsed with isopropyl alcohol. Observation in an optical light microscope revealed that the sample surfaces were not smeared or otherwise deformed, which could influence the hardness test results (Fig. 1). The test surfaces were smooth, even, and free from oxide scale, foreign matter, and lubricants, conforming to Sect. 6.1 of ASTM E18-11 [1].

A digital Rockwell hardness tester [9] was used to create and measure ten hardness indentations on each specimen surface with a spheroconical diamond indenter tip. Prior to the data acquisition, the hardness tester was calibrated and verified according to Sect. A1.5 of ASTM E18-11 [1]. All of the hardness tests were performed on the same day by the same operator on the same hardness tester, in order to use $r_{\mathrm{PB}}$ as an analysis reference. The anvil, indenter, and samples were all cleaned with ethyl alcohol and dried using a lint-free wipe and compressed air spray. For these specimens, the HRC scale was used with a load of $150 \mathrm{kgf}$, conforming to ASTM E18-11 for medium carbon steel samples [1]. The dwell time for the preliminary force was 


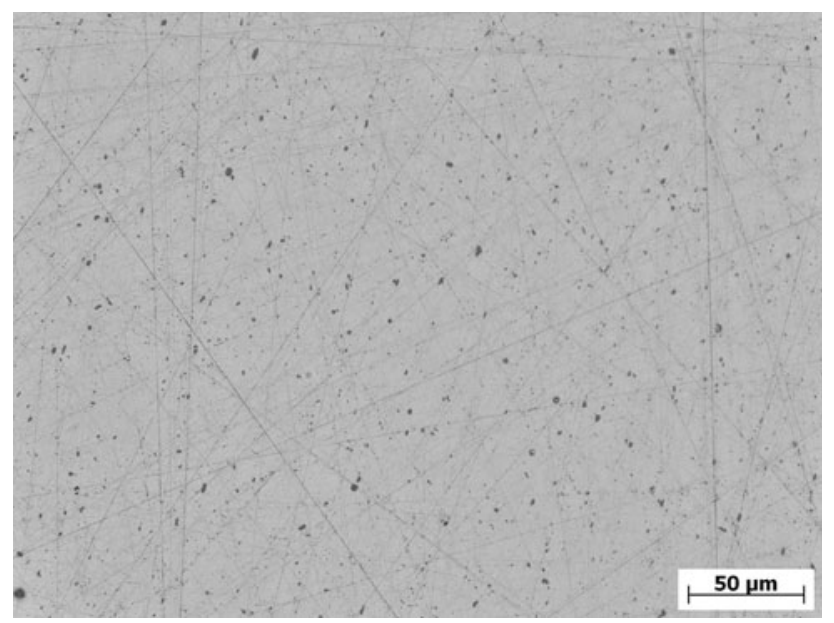

Fig. 11144 Steel sample, taken with the $\times 50$ objective on an optical light microscope after the $3-\mu \mathrm{m}$ polishing step, showing appropriate sized scratches and some inclusions

$3 \mathrm{~s}$, and the dwell time for the total force was $4 \mathrm{~s}$. This specific model hardness tester has an automatic stop, automatic loading and dwelling, and automatic Rockwell hardness calculations, helping to ensure that the tests were as consistent as possible.

The temperature of the room was $20{ }^{\circ} \mathrm{C}$ during the tests, with fluctuations of no more than $\pm 1{ }^{\circ} \mathrm{C}$, and there was a $40 \%$ humidity level. All factors throughout the entire experiment were controlled as tightly as possible to reduce the amount of measurement error. The results were calculated directly from the depth measurements to the Rockwell $\mathrm{C}$ scale by the hardness tester; no conversion charts were used.

\section{Results and Analysis}

With five specimens per mounting material and ten hardness indentations per specimen, there were 50 data points collected for each test group. The average and standard deviation values were then calculated for each group (Table 1).

The control group had the lowest standard deviation. Specimens encapsulated in green phenolic and transparent thermoplastic had the highest standard deviations; these mounting materials are likely not as hard as the others, allowing the specimens to shift more during the Rockwell hardness tests.

An $r_{\mathrm{PB}}$ of $0.937 \mathrm{HRC}$ was found for a $25.0 \mathrm{HRC}$ test block from ASTM RR:E28-1021 [5]. As long as two test results are within 0.937 HRC of each other, there is approximately a $95 \%$ probability that they are equivalent. It is assumed that the repeatability $(r)$ of this study would not vary from $r_{\mathrm{PB}}$. Further study, including a complete evaluation of measurement uncertainty, would be necessary to calculate $r$ for this specific set of conditions. The green phenolic and transparent thermoplastic test groups had standard deviations that were greater than $r_{\mathrm{PB}}$, meaning that enough data points collected for each test group were outside the range to which they would be considered equivalent. Therefore, the green phenolic and transparent thermoplastic mounting materials do not provide adequate support for Rockwell hardness testing.

The averages and standard deviations were plotted for easier comparison (Fig. 2). The mounting materials listed across the $x$ axis in Fig. 2 are in no particular order; a follow-up study could be performed to see if there is any correlation between the hardness of the mounting materials and the hardness results of the specimens.

Table 1 Averages and standard deviations for Rockwell hardness test values of encapsulated and unencapsulated metallographic specimens

\begin{tabular}{lll}
\hline Mounting material & $\begin{array}{l}\text { Average hardness } \\
(\text { HRC })^{\mathrm{a}}\end{array}$ & $\begin{array}{l}\text { Standard deviation } \\
(\text { HRC })^{\mathrm{b}}\end{array}$ \\
\hline Green phenolic & 23.70 & 1.34 \\
Orange phenolic & 24.37 & 0.73 \\
$\begin{array}{l}\text { Black glass-filled } \\
\text { epoxy }\end{array}$ & 23.94 & 0.78 \\
$\quad$ Transparent & 23.00 & 1.08 \\
$\quad$ thermoplastic & & \\
QuickCure acrylic & 24.07 & 0.42 \\
EpoxySet epoxy & 24.29 & 0.64 \\
No Mounting-control & 24.03 & 0.38 \\
group & &
\end{tabular}

${ }^{a}$ Calculated using the AVERAGE function in Microsoft Excel 2003

b Calculated using the STDEV function in Microsoft Excel 2003

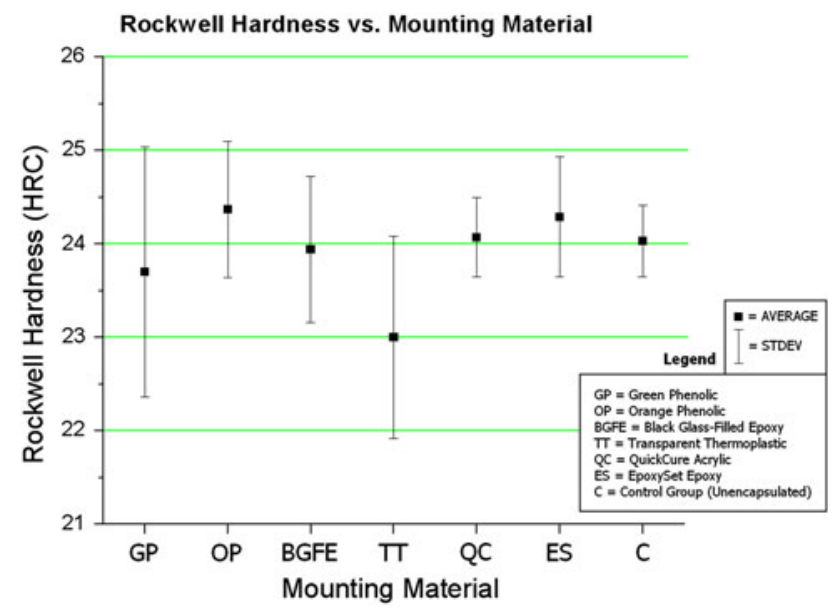

Fig. 2 Plot of each test group's Rockwell hardness value averages (square points) and standard deviations (error bars) 
Fig. 3 Line plot of each test group's average Rockwell hardness value (HRC) compared to the $-r_{\mathrm{PB}}$ and $+r_{\mathrm{PB}}$ values
Rockwell Hardness (HRC)

(Increasing from left to right)

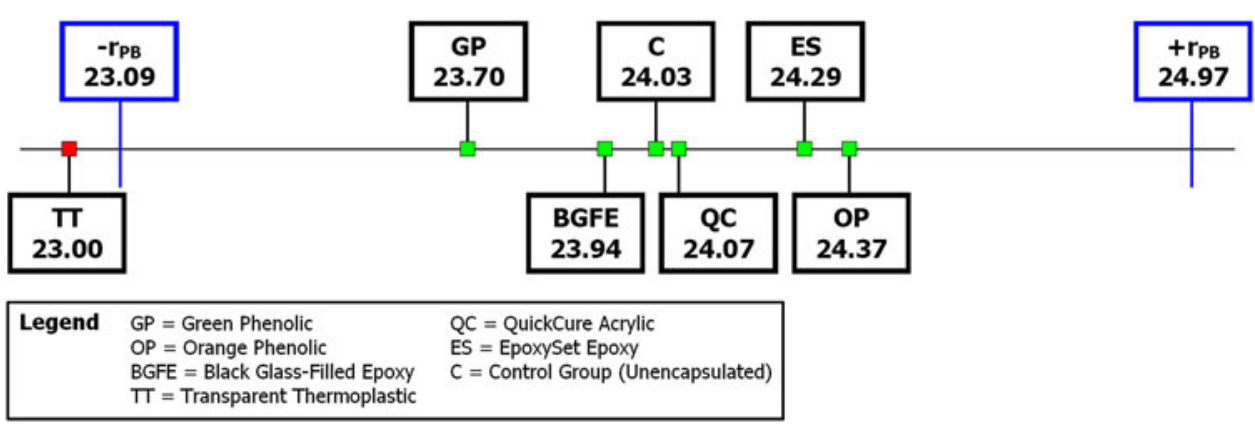

All the mounting materials except for the transparent thermoplastic fell within 0.937 HRC of the control group average of 24.03 HRC. For easier comparison of each test group's average hardness to the $r_{\mathrm{PB}}$ range, the data was plotted in ascending order, in a single line (Fig. 3).

Not only was the transparent thermoplastic inconsistent due to its large standard deviation, but also it had a very low probability of being equivalent to the control group due to it falling outside the $r_{\mathrm{PB}}$ range. All of the other mounting materials had Rockwell hardness values clustered close to the control group. Some of the hardness test values for transparent thermoplastic fell within the $r_{\mathrm{PB}}$ range, so those may be considered equivalent. However, enough of the individual points fell outside the range that the average hardness value was pushed lower than $-r_{\mathrm{PB}}$.

\section{Conclusions}

Five out of the six test groups (all except transparent thermoplastic) had average hardness values that fell within $\pm r_{\mathrm{PB}}$ ( $\left.\pm 0.937 \mathrm{HRC}\right)$ of the control group average of 24.03 $\mathrm{HRC}$, so it is approximately $95 \%$ probable that those hardness values are equivalent to the control group. Four of the six test groups (all except green phenolic and transparent thermoplastic) had standard deviations that were less than $r_{\mathrm{PB}}$. With such results, it is reasonable to say that green phenolic and transparent thermoplastic are not suitable mounting materials for Rockwell hardness testing. Orange phenolic, black glass-filled epoxy, QuickCure acrylic, and EpoxySet epoxy [7] may all be adequate for supporting specimens during Rockwell hardness testing. Further study would be necessary; however, this is contrary to common practices that recommend not performing Rockwell hardness tests on encapsulated specimens [2].

Factors that could influence the results further and cause the hardness test values to fall outside the $r_{\mathrm{PB}}$ range include the cross-sectional area of the specimen, the expected hardness of the specimen, inconsistent metallographic preparation, and fluctuations in the curing of the mounting materials. A complete evaluation of measurement uncertainty would also be necessary to validate results and calculate $r$ for the specific set of conditions in this study.

With all those factors adding to the uncertainty, some follow-up tests could be performed to reduce such uncertainty. This could include taking sample material from a certified Rockwell hardness test block, testing different mount curing parameters, testing multiple cross-sectional surface area sizes, and performing these tests on materials of different hardness values. This study focused on a grade 1144 steel rod, so other types of materials could also be tested. Perhaps the results could be normalized or interpreted differently if Shore durometer hardness tests are also performed on the cured mounting materials themselves. Depending on the results from follow-up studies, ASTM E18 may need to be revised accordingly.

Acknowledgments Clayton A. Smith, Allied High Tech Products, Inc.; Gary Liechty, Allied High Tech Products, Inc.; Edward Hirsch, Allied High Tech Products, Inc.; Kim Dermit, Allied High Tech Products, Inc.; Chris Richardson, Allied High Tech Products, Inc.; Sunny Hung-Hsun Wei, Allied High Tech Products, Inc.; Frank J. Morales, Allied High Tech Products, Inc.

\section{References}

1. ASTM International Standard E18, Standard Test Methods for Rockwell Hardness of Metallic Materials, ASTM International, West Conshohocken, PA, 2011. doi:10.1520/E0018-11, www. astm.org

2. Tips and Rules About Hardness Testing, Struers, Inc., www. struers.com. Accessed January 2013

3. ASTM International Standard D2240, Standard Test Method for Rubber Property-Durometer Hardness, ASTM International, West Conshohocken, PA, 2010, doi:10.1520/D2240-05R10, www.astm.org

4. Durometer Testing, Corporate Consulting, Service \& Instruments, Inc., http://www.ccsi-inc.com/t-durometer-testing.pdf. Accessed May 2013

5. ASTM International Research Report RR:E28-1021, Inter-Laboratory Study to Establish Precision Statements for ASTM E18, p. 3. ASTM International, West Conshohocken, PA, 2004, www.astm.org 
6. High-Strength Easy-to-Machine 1144 Carbon Steel, McMasterCarr, www.mcmaster.com. Accessed Dec 2012

7. Mounting Products, Allied High Tech Products, Inc., www. alliedhightech.com. Accessed May 2013
8. Polishing Cloths, Allied High Tech Products, Inc., www. alliedhightech.com. Accessed May 2013

9. HR-210MR/320MS/430MR/430MS Series 963-Rockwell Hardness, Mitutoyo America Corp., www.mitutoyo.com. Accessed May 2013 